Ilchmann, Achim; Mareels, Iven M. Y.; Van Gils, S.; Poldermann, J.W. :

Asymptotic Dynamics in Adaptive Gain Control

Aus:

Advances in Control, Highlights of ECC '99, Ed.: P.M. Frank [European Control Conference ; 5 (Karlsruhe) : 1999.08.31-09.03], London : Springer-Verlag, 1999. - ISBN 1-85233-122-4, S. 29-63 


\title{
2
}

\section{Asymptotic Dynamics in Adaptive Gain Control}

\author{
I. Mareels, S. Van Gils, J. W. Polderman and A. Ilchmann
}

Department of Electrical and Electronic Enginecring, The University of Melbourne, Vic 3052, Australia, i.mareels@unimelb.edu.au

Faculty of Mathematical Sciences, The University of Twente, Enschede, The Netherlands, s.a.vangils@math.utwente.nl

Faculty of Mathematical Sciences, The University of Twente, Enschede, The Netherlands, $j$.w.polderman@math.utwente.nl

School of Mathematical Sciences, University of Exeter, Exeter EX4 4QE, UK, ilchmann@maths . exeter.uk

Summary. It is well known that linear SISO systems that can be rendered passive through constant output feedback can be adaptively stabilised through a single gain adaptation law. We revisit the dynamical bchaviour of such systems and exhibit through a bifurcation analysis a rich variety of potcntial asymptotic dynamics, for which we provide a control theoretic interpretation. This in turn leads us to question the actual adaptive control question and solution approach.

\section{Introduction}

Adaptive output gain control for the purposes of regulation and without reliance on identification or probing signals constitutes about the simplest adaptive control objective one can consider. Research into this idea was initiated by Mareels [3], Morse [12], and further developed by Willems and Byrnes [10]. For an overvicw and extensive reference list see Ilchmann [4]. The work of 
Willems and Byrnes lead to the whole topic of universal controllers see e.g. [7] and [2, Chapter 6]. The topic of adaptive stabilisation via output feedback is also studied in great detail by [11]. In this paper we revisit this adaptive scheme from a dynamical systems perspective. Our aim is to understand the adaptive control question better and to be in a position to recast adaptive control questions in a more appropriate context.

In order to apply adaptive output gain control one starts from the assumption that the plant to be controlled is a finite dimensional SISO linear system that can be stabilised by static high gain output feedback. Adaptation is required because the amount of gain in order to achieve regulation is unknown. A large class of linear systems that can be stabilised by output gain feedback is characterised by: the plant's transfer function has stable zeroes and relative degree one. Such systems can be conveniently represented as follows [2, Section 6.4] (see also the Appendix I):

$$
\begin{aligned}
\dot{x} & =A x+b y, \\
\dot{y} & =-c x-d y+g u .
\end{aligned}
$$

Here $u$ is the input, $y$ the output and $(x, y) \in \mathbb{R}^{n} \times \mathbb{R}$ is the state of the linear systcm (1). $A \in \mathbb{R}^{n \times n}$ is a matrix whose eigenvalues have negative real part. The eigenvalues of the matrix $A$ are precisely the zeroes of the transfer function of the system (1). We assume that $g>0, g$ is the high frequency gain from input to output. We assume that the original system (1) is controllable. As a consequence, see Appendix I, the pair $(A, b)$ is controllable. It is clear that for all sufficiently large $k>0$, the output feedback control law $u=-k y$ stabilises the system (1), sec e.g. $[3,5]$.

In this paper we are interested in characterising the qualitative behaviour (the phase portrait) when the adaptive feedback law

$$
\begin{aligned}
& u=-k y+e, \\
& \dot{k}=-\sigma k+y^{2}, \quad k(0)>0 .
\end{aligned}
$$

is applied to (1). Here $\sigma \geq 0$ is a small positive constant representing the so called sigma modification [8] and $e \in \mathbb{R}$ represents a control offset error. The control offset error is introduced to unearth some of the robustness issues of concern in adaptive control. One could object that a constant offset error is not a realistic disturbance as it is casily overcome via integral control action. As is shown in Appendix V, this is false, and the discussion we present remains relevant even if integral action is explicitly taken into account.

For $\sigma=0$ and $e=0$ the intuition behind the adaptation law is simple. As long as $y \neq 0$ the feedback gain $k$ increases, eventually rendering the $(x, y)$ subsystem asymptotically stable. The output will decrease to zero in an exponential manner which limits the feedback gain. Unfortunately, any nonzero measurement crror or disturbance such as $e$ will force the feedback gain to grow indefinitely. 
This observation of lack of robustness lead to the so called sigma modification. This idea was first suggested by Ioannou and Kokotovic [8] in the context of adaptive model reference control. It was adopted for systems of the form (1) by Bar-Kana and co-workers, see [11] for a detailed overview of this work. Another possible modification to overcome this lack of robustness problem whilst preserving the simplicity of the controller is the so called $\lambda$-tracking approach (first suggested in [3] and introduced by Ilchmann and Ryan [6], see also Ilchmann [5]). In this line of work a dead-zone in the gain adaptation is invoked. The dead-zone reduces the performance of regulation in the disturbance free case in that the output converges to a prespecified, $\lambda$ neighbourhood of the reference signal. This is a small price to pay for the added robustness with respect to small disturbances. The $\lambda$-tracking modification will not be considered in the present paper.

Compared to the above simple intuitive picture, the sigma modification alters the phase portrait in a non trivial way. This is the topic studied in this paper. The prime purpose of the sigma modification is to obtain robustness with respect to measurement errors. This is indeed achieved, but at a price, as the asymptotic dynamies are no longer as desired. We investigate this phenomenon in some detail, suggesting some alternatives.

Despite the rather large literature on the topic it is our contention that our understanding of the dynamics at large of these simple adaptive control systems is not complete. In this paper we collect a number of results describing what can be expected both for the transients as well asymptotic behaviour. Moreover describing these dynamics leads us to an understanding of relevant adaptive control questions.

The paper is organised as follows, in the preamble following the introduction, we gather a number of known results and introduce some notation. The next section is devoted to the ultimate boundedness that the adaptively controlled system dynamics enjoy. The results established here serve to underscore the importance of the sigma modification. Then we discuss the simplest possible scenario, when the linear system to be controlled has no zeroes. This situation is actually simpler than what (1) suggests, but nevertheless informative. This leads to a planar adaptive system, whose dynamics can be well understood. It provides a good pointer for the complications we may expect. Next follows an incomplete analysis for the case when the linear system to be controlled has a single stable zero and two poles. Finally we conjecture what a phase portrait in the general case may look like. The paper concludes with suggestions on how we may have to modify the adaptive law based on the understanding of the exposed dynamical behaviour in order to obtain a robust adaptive system.

\section{Preamble}

For convenience sake and without loss of generality (as far as the dynamical system analysis is concerned) we study the closed-loop system (1), (2) for 
- $g=1$, if $g \neq 1$ consider the state space transformation $(x, y, k) \rightarrow$ $(x \sqrt{g}, y \sqrt{g}, k / g)$,

- $(A, b)$ in controllable canonical form.

The closed-loop adaptive system can thus be represented as:

$$
\begin{array}{ll}
\dot{x}=A x+b y, & x(0) \in \mathbb{R}^{n}, \quad b \in \mathbb{R}^{n}, A \in \mathbb{R}^{n \times n} \text { stable, } \\
\dot{y}=-c x-d y-k y+e, & y(0) \in \mathbb{R}, \quad c^{T} \in \mathbb{R}^{n}, d, e \in \mathbb{R}, \\
\dot{k}=-\sigma k+y^{2}, & k(0) \geq 0, \quad \sigma \geq 0 .
\end{array}
$$

We refer to the subsystem with state $(x, y)$ as the plant. We consider the parameters $e, d$ and the vector $c$ as bifurcation parameters.

We use the notation:

$$
\begin{aligned}
& z(s)=\operatorname{det}(s I-A) \\
& p(s)=\operatorname{det}\left(\begin{array}{cc}
s I-A & -b \\
c & s
\end{array}\right) .
\end{aligned}
$$

Notice that with the above notation:

$$
\operatorname{det}\left(\begin{array}{cc}
s I-A & -b \\
c & s+\beta
\end{array}\right)=p(s)+\beta z(s) .
$$

We conclude this section with some simple observations about the phase portrait of the system (3):

- Fact (i) $k(t) \geq 0$ for all $t \geq 0$ if $k(0) \geq 0$.

- Fact (ii)

If $e=0,(x(t), y(t), k(t))$ is a solution of $(3)$ if $(-x(t),-y(t), k(t))$ is one.

- Fact (iii)

For $\sigma>0, e=0$ and $(x(0), y(0))=(0,0)$, it follows that $k(t)=e^{-\sigma t} k(0)$ and $(x(t), y(t))=(0,0)$ for all $t \geq 0$.

\section{- Fact (iv)}

For $\sigma=0$ and $e=0$, the trajectories converge to a point in state space of the form $\left(0,0, k_{\infty}\right)$, for some $k_{\infty} \geq 0$ which depends on the initial condition. (See [2]).

- Fact (v)

For $\sigma=0$ and $e \neq 0,(x(t), y(t))$ converge to zero and $k(t)$ diverges.

- Fact (vi)

The transformation $(x, y, k)=\sigma\left(x_{1}, y_{1}, k_{1}\right)$ and time scaling $\sigma t=\tau$, 
leads to a systom description of the form:

$$
\begin{aligned}
x_{1}^{\prime} & =A_{1} x_{1}+b_{1} y_{1}, \\
y_{1}^{\prime} & =-c_{1} x_{1}-d_{1} y-k_{1} y_{1}+e_{1}, \\
k_{1}^{\prime} & =-k_{1}+y_{1}^{2} .
\end{aligned}
$$

Here $\sigma A_{1}=A, \sigma b_{1}=b, \sigma c_{1}=c, \sigma d_{1}=d$ and $\sigma^{2} e_{1}=e$. The ' denotes derivative with respect to the new time variable $\tau$. Clearly (5) is of the same form as (3). Hence, when discussing the dynamics we can limit ourselves to the case $\sigma=1$. Yet, from a control perspective, we prefer (3), as $\sigma$ is a design variable and $A, b, c, d$ are not.

\section{Global stability}

It is clear from Fact (iv) and Fact (v) that the adaptive system with $\sigma=$ 0 is not robust with respect to input offsets. The sigma modification has the property that all trajectories are ultimately bounded ${ }^{1}$, regardless of the disturbance $e$. This is probably the main motivation for its introduction. The ultimate boundedness result can be established using a Lyapunov argument.

Theorem 3.1 Consider (3) with $\sigma>0$. Let $P=P^{T}>0$ be the unique positive definite solution of the Lyapunov equation $A^{T} P+P A=-I$. For all $\sigma<\bar{\sigma}=1 / \lambda_{\max }(P)$ the compact set

$$
\mathbb{A}=\left\{(x, y, k): x^{T} P x+y^{2}+(k-\mu)^{2} \leq \mu^{2}+|e|\right\},
$$

with

$$
\mu=\max \left\{0,-d+\frac{1}{2}\left(\frac{|e|}{2 \sigma}+\sigma+\left(P b-c^{T}\right)^{T}(I-\sigma P)^{-1}\left(P b-c^{T}\right)\right)\right\}
$$

is globally attractive and positively invariant.

Proof Consider the comparison function

$$
V(x, y, k)=x^{T} P x+y^{2}+(k-\mu)^{2} .
$$

Its derivative along the solutions of system (3) is given by:

$$
\begin{aligned}
\dot{V}(x(t), y(t), k(t))= & -x^{T} x+2 x^{T} P b y-2 x^{T} c^{T} y-2 d y^{2}-2 k y^{2}+2 y e \\
& -2 \sigma k(k-\mu)+2(k-\mu) y^{2}, \\
= & -x^{T} x+2 x^{T} P b y-2 x^{T} c^{T} y-2 d y^{2}+2 y e-2 \mu y^{2} \\
& -2 \sigma(k-\mu)^{2}+2 \sigma \mu^{2} .
\end{aligned}
$$

\footnotetext{
${ }^{1}$ The solutions of $\dot{x}=f(x)$ are said to be ultimately bounded, if there exists a $B>0$ such that for all initial conditions $x(0)=x_{0}$ there exists a $T\left(x_{0}\right)>0$ such that the solutions satisfy $\left\|x\left(t, x_{0}\right)\right\| \leq B$ for all $t \geq T$.
} 
Selecting $\mu$ as indicated in (7) implies that

$$
-x^{T} x+2 x^{T} P b y-2 x^{T} c^{T} y-2 d y^{2}+2 y e-2 \mu y^{2} \leq-\sigma\left(x^{T} P x+y^{2}\right)+2 \sigma|e|,
$$

which leads to:

$$
\dot{V}(x(t), y(t), k(t)) \leq-2 \sigma V(x(t), y(t), k(t))+2 \sigma \mu^{2}+2 \sigma|e|
$$

This establishes our claim.

Remark 3.2 The theorem statement is particularly useful for the case that $\sigma$ is small. It is clear from the proof that ultimate boundedness can be established for all $\sigma>0$, but we prefer the above formulation as it provides us with a reasonably tight estimate of the form $V(x, y, k) \leq \mu^{2}+|e|$ for a positively invariant set. In case $e=0$ our estimate cannot be improved as the set $\mathbb{A}$ has the origin (an equilibrium in this case) on its boundary. Moreover, "small" $\sigma$ is in line with practice.

For $e=0$ we can glean from the theorem statement that for sufficiently large, positive $d$ the origin of the state space is globally asymptotically stable. It can be established that this is the case for all systems (1) that have strict positive real transfor function, (i.e. $\operatorname{Re}\{z(j \omega) /[p(j \omega)+d z(j \omega)]\}>0$ for all $\omega \in \mathbb{R})$.

Theorem 3.3 Consider system (3) with $\sigma>0$ and $e=0$. Assume furthermore that the linear system (1) has a strictly positive real transfer function, i.e. there exists a positive definite solution $P=P^{T}$ such that $A^{T} P+P A<$ 0 and $d>\left\|c^{T}-P b\right\|^{2}$. Then the origin of the state space is globally uniformly asymptotically stable.

Proof The result is derived using the comparison function $V$ introduced in equation (8) with $\mu=0$. The conditions of the Theorom 3.3 lead to $\dot{V}(x(t), y(t), k(t))<-2 \sigma k^{2}(t)-W(x(t), y(t))$, with positive definite $W(x, y)$.

The gap between the two results stated in Theorem 3.3 and 3.1 does not appear to be large. One could wonder whether the same result as stated in Theorem 3.3 could hold true for the larger class of stable minimum phase systems. This is not the case, as will become clear. Here is a simulation example to illustrate what can happen.

Consider a system of the form (3) with the parameters selected as:

$$
A=\left(\begin{array}{cc}
0 & 1 \\
-1 & -10
\end{array}\right) \quad b=\left(\begin{array}{l}
0 \\
1
\end{array}\right) \quad c=\left(\begin{array}{cc}
-9 & -10
\end{array}\right) \quad d=-1 \quad \sigma=0.1
$$

The linear system (1), with the above parameter values, has a transfer function with stable zeroes and stable poles. The transfer function is given by $z(s) /(p(s)+d z(s))=\left(s^{2}+s+10\right) /\left(s^{3}+2 s^{2}+s+1\right)$. This transfer function is not strictly positive real. A typical trajectory for the above system 
with initial conditions $(0.1,0.1,1,0)$ is presented in Figure 1. Both the plant output $y$ and the adaptive gain $k$ are displayed. Notice the asymptotically periodic behaviour. A root locus plot for that root of $p(s)+(d+k) z(s)=$ $s^{3}+3 s^{2}+2 s+11+(-1+k)\left(s^{2}+s+10\right)$ with the largest real and largest imaginary part is presented in Figure 2. Observe that the adaptive gain hovers asymptotically around that value of the gain above which the root locus remains in the left half plane, clearly the plant is but marginally stabilised through the adaptive control law. Observe that no control would have been better in this case, as the open loop system is stable.
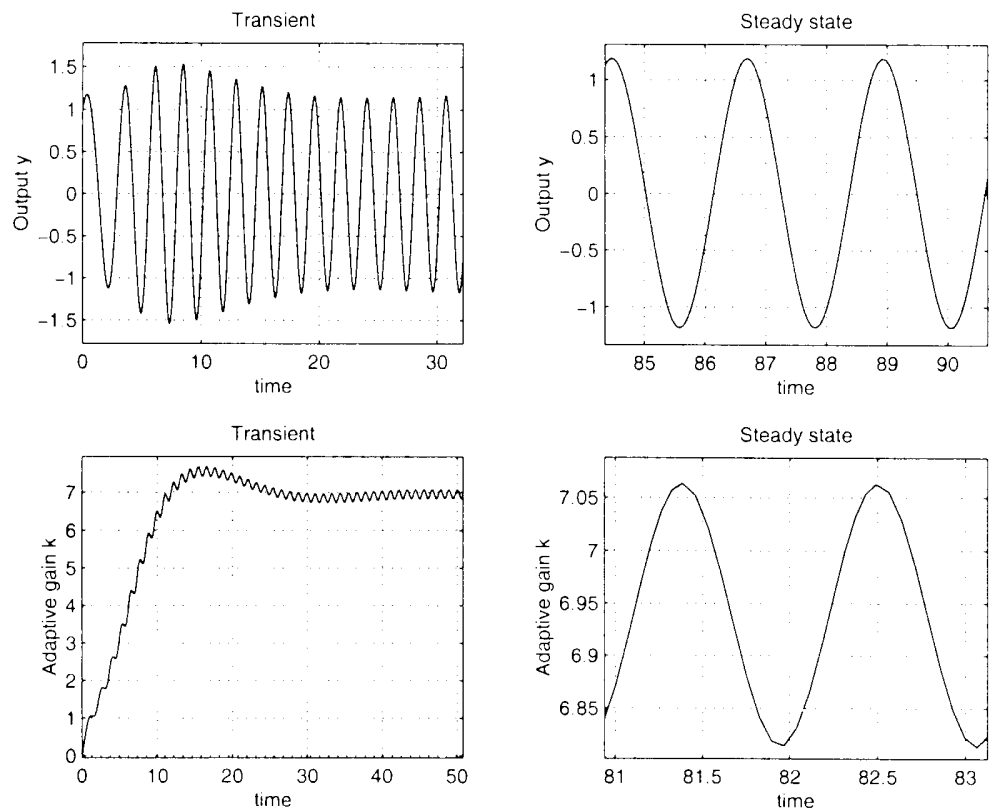

Figure 1: Limit cycle behaviour for adaptively controlled stable minimum phase plant

\section{Planar adaptive system}

Let us first consider the adaptive stabilisation through adaptive gain output feedback of a one dimensional linear plant:

$$
\begin{array}{ll}
\dot{y}=-d y-k y+e, \quad y(0), \\
\dot{k}=-\sigma k+y^{2}, \quad k(0) \geq 0 .
\end{array}
$$

Here $d$ is the open loop pole of the plant to be stabilised, $y$ is the measured output, $k$ the adaptive feedback gain and $e$ represents the input disturbance. 


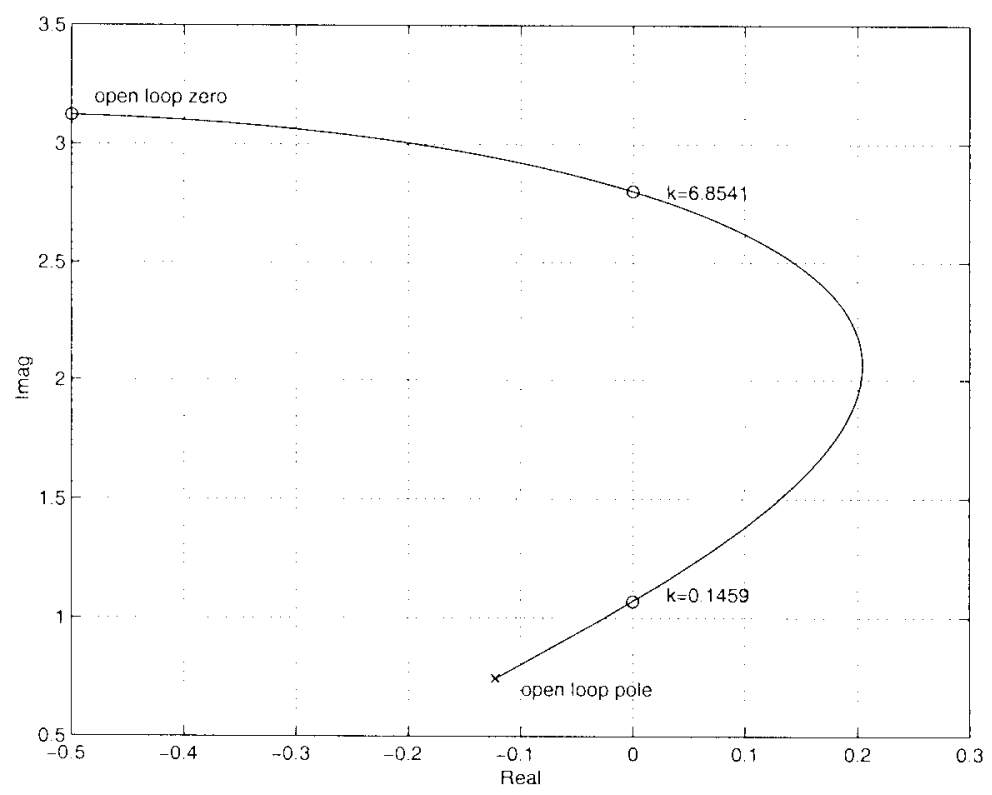

Figure 2: Partial root locus for stable minimum phase plant

For purposes of comparison, if we had known the parameter $d$, then any control gain such that $k+d>0$ yields a stable system with equilibrium $y_{e}=$ $e /(k+d)$. For control performance we would presumably require $\left|y_{e}\right|<|e|$, hence $k+d>1$.

\subsection{Unperturbed adaptive system}

For the adaptive system (9) with $e=0$, we can make the following obscrvations:

1. An open loop stable plant $d \geq 0$ implies that the trivial solution is globally asymptotically stable.

Clearly the solution $(y(t), k(t))=(0,0)$ is uniformly asymptotically stablc as scen from considering the Lyapunov function $V(y, k)=y^{2}+k^{2}$. For the derivative of $V$ along the solutions of (9) wo obtain $\dot{V}(t)=$ $-2 d y^{2}(t)-2 \sigma k^{2}(t)$. It follows that the origin is globally asymptotically stable. This observation is also a corollary of Theorem 3.3, as any linear stable first order system has a strictly positive real transfer function.

2. An open loop unstable plant $d<0$ leads to solutions that are ultimately bounded

Consider the Lyapunov function $V(y, k)=y^{2}+(k+d-\sigma)^{2}$. We obtain for the derivative along the solutions of $(9)$ that $\dot{V}(y(t), k(t))=$ 
$-2 \sigma y^{2}(t)-2 \sigma k^{2}(t)+2 \sigma(-d+\sigma) k(t)$. Considering that $k(t) \geq 0$, we obtain the estimate $\dot{V}(y(t), k(t)) \leq-2 \sigma V(y(t), k(t))+2 \sigma(-\bar{d}+\sigma)^{2}$. From which it follows that the set characterised by $V(y, k) \leq(-d+\sigma)^{2}$ is positively invariant. This observation is also a corollary of Theorem 3.1 .

3. For an open loop unstable plant $d<0$ the solutions either converge to $(\sqrt{-\sigma d},-d)$ or $(-\sqrt{-\sigma d},-d)$, unless $y(0)=0$ in which case the solutions converge to $(0,0)$.

If $y(0)=0$ it follows that $y(t)=0$ for all $t \geq 0$ and furthermore that $k(t)=\exp (-\sigma t) k(0)$. If $y(0)>0$ then obviously $y(t)>0$ for all $t \geq 0$. Consider the Lyapunov function $V(y, k)=y^{2}-2 \sigma d \ln y+(k+d)^{2}$ on the domain $D=\{y>0, k>0\} . V$ achieves its minimum in $(\sqrt{-\sigma d},-d)$. For its derivative along the solutions of $(9)$ we find $\dot{V}(y(t), k(t))=$ $-2 \sigma(k(t)+d)^{2}$. Applying LaSalle's invariance principle, it follows that all solutions starting in $D$ converge to the point $(\sqrt{-\sigma d},-d)$. This cstablishes our claim, as the situation $y(0)<0$ can be dealt with in an analogous manner.

The above describes a single pitch fork bifurcation for the origin attained at $d=0$. The typical local stability picture associated with this bifurcation has global validity in this particular case. For $d>0$ the origin is globally asymptotically stable, for $d<0$ the origin becomes a saddle and two new locally stable equilibria come into existence. In this particular situation the stable manifold of the saddle (the origin) acts as a separatrix between the domains of attraction for the other equilibria.

The control interpretation is as follows. In the absence of any disturbances, the adaptive sigma modificd control law adjust the feedback gain $k$ to the minimum feedback action required to stabilise the open loop plant. Indeed, if the open loop plant was stable, asymptotically no feedback is applied. If the open loop plant was unstable, the minimum feedback gain to render the lincar plant marginally stable is applied. In doing so the adaptive control law sacrifices the control objective of achieving regulation of the output $y$, instcad $|y|$ converges to $\sqrt{-\sigma d}$. For small values of $\sigma$ (meaning $\sigma<<1 / d$ ) this loss of asymptotic performance may be acceptable. It is nevertheless disappointing. In this particular case of a first order system we know a priori that arbitrary transient performance can be achieved for correspondingly large gains. The adaptive law however limits the gain to the minimum gain required to just, achieve stability.

\subsection{Perturbed adaptive system}

It is disturbing to notice that the adaptive law leads to a marginally stabilised linear plant in the case the latter was open loop unstable. This is the more annoying when we realise that this is the only case in which some control is 
required and that good performance can be achieved via high feedback gain. What happens if measurement errors are involved, i.e. $e \neq 0$ ?

Importantly, $e \neq 0$ breaks the symmetry inherent in (9) when $e=0$, hence the pitchfork bifurcation pattern breaks as $e \neq 0$. Moreover in view of the fact we now have a two parameter family of planar vector fields ( $e, d$ are the important parameters, $\sigma$ is not) we expect that we can organise the local dynamics around a Takens-Bogdanov bifurcation point. This would lead to the presence of a homoclinic bifurcation and a Hopf bifurcation in the bifurcation diagram. A bifurcation diagram is presented which describes the local dynamics. For the transients we offer some analytic results, but mainly rely on simulation results.

1. For $d \geq 0, e \neq 0$ there is a unique globally asymptotically stable equilibrium.

The equilibrium is defined via $\sigma k_{e}=y_{e}^{2}$ and $y_{e}^{3}+\sigma d y_{e}=\sigma e$. With $d \geq 0$ the lattcr equation implies that the equilibrium exists and is unique. Local stability follows at once from the Jacobian:

$$
J=\left(\begin{array}{cc}
-d-k & -y \\
2 y & -\sigma
\end{array}\right) .
$$

The characteristic polynomial of $J$ at an equilibrium $\left(y_{e}, k_{e}\right)$ is given by $\lambda^{2}+\left(d+k_{e}+\sigma\right) \lambda+\sigma d+3 y_{e}^{2}$. Using the fact that $y_{e}^{2}=\sigma k_{e}$ at equilibrium, this can be rewritten in the more informative format

$$
\operatorname{det}(\lambda I-J)=(\lambda+d)(\lambda+\sigma)+k_{e}(\lambda+3 \sigma) .
$$

Global stability can be established using a Lyapunov argument. Consider the case $e>0$. Let $V(y, k)=\left(y-y_{e}\right)^{2}+\left(k-k_{e}\right)^{2}$. Observe that the set $\mathbb{D}=\{(y, k): y \geq 0, k \geq 0\}$ is attractive and positively invariant. On $\mathbb{D}$ we have $V(y(t), k(t)) \leq-\sigma\left(k-k_{e}\right)^{2}-\left(d+k_{e}\right)\left(y-y_{e}\right)^{2}$.

2. For $d<0$ and $|e|>e_{S N}$, with $e_{S N}=\frac{2}{3} \sqrt{\frac{\sigma}{3}}(-d)^{3 / 2}$ there is a unique, locally stable equilibrium.

Equilibria satisfy $\sigma k_{e}=y_{e}^{2}$ and $y_{e}^{3}+\sigma d y_{e}=\sigma e$. With $d<0$ and $|e|>e_{S N}$ the latter equation implies that the equilibrium exists and is unique.

Local stability follows at once from the Jacobian (10). As under the given circumstances $y_{e}^{2}>-\sigma d$ or $k_{e}>-d$ it follows that $d+k_{e}+\sigma>$ $\sigma>0$ and $\sigma\left(d+3 k_{e}\right)>-2 \sigma d>0$, which by inspection of (11) implies that $\left(y_{e}, k_{e}\right)$ is locally asymptotically stable.

3. For $d<0$ and $|e| \leq e_{S N}$, there are three equilibria, $\left(y_{e i}, \sigma y_{e i}^{2}\right)$ $i=1,2,3$ with $-y_{S N} \leq y_{e 1} \leq-\sqrt{\frac{-\sigma d}{3}} \leq y_{e 2} \leq \sqrt{\frac{-\sigma d}{3}} \leq y_{e 3} \leq y_{S N}$, where $y_{S N}$ is the positive root of $y^{3}+\sigma d y=\sigma e_{S N}$. 
The locus

$$
e^{2}=\frac{4}{27} \sigma(-d)^{3}
$$

describes the Saddle-Node bifurcation in the bifurcation diagram.

The equilibrium $\left(y_{e 2}, \sigma y_{e 2}^{2}\right)$ is an unstable saddle. Indeed the product of the eigenvalues of the Jacobian evaluated at this equilibrium is given by $\sigma d+3 y_{e 2}^{2} \leq 0$. The equilibria for $i=1,3$ may be stable or unstable depending on the values of $d, e$. If $\sigma+2 d / 3>0$ they are locally stable, regardless of the value of $e$. They change their stability status through a Hopf bifurcation if $\sigma+2 d / 3<0$. When $e$ is less than but near $e_{S N}$ the equilibrium $i=3$ is locally stable, whilst the equilibrium $i=1$ is unstable.

The Hopf bifurcation locus in the bifurcation diagram is described by:

$$
e^{2}=-\sigma^{3} d-\sigma^{4} \text { with } d<-3 / 2 \sigma .
$$

The equilibria undergoing the Hopf bifurcation are described by:

$$
\begin{aligned}
& y_{e}^{2}=\sigma(d+\sigma)^{2}, \quad y_{e} e<0, \\
& k_{e}=-d-\sigma .
\end{aligned}
$$

\section{Takens-Bogdanov bifurcation point}

In order to organise the bifurcation diagram more clearly, we investigate the presence of a Takens-Bogdanov bifurcation point and verify its local dynamics. In view of the fact that we are dealing with a two-parameter family of vector ficlds we expect to find some isolated Takens-Bogdanov points. The calculations used to obtain the normal form for the unfolding are summarized in Appendix II.

The Takens-Bogdanov points for (9) are given by:

$$
y_{T B 1}=\frac{\sigma}{\sqrt{2}}, \quad k_{T B}=\frac{\sigma}{2}, \quad d_{T B}=-\frac{3 \sigma}{2}, \quad e_{T B 1}=-\frac{\sigma^{2}}{\sqrt{2}},
$$

and

$$
y_{T B 2}=-\frac{\sigma}{\sqrt{2}}, \quad k_{T B}=\frac{\sigma}{2}, \quad d_{T B}=-\frac{3 \sigma}{2}, \quad e_{T B 2}=\frac{\sigma^{2}}{\sqrt{2}} .
$$

As is easily verified, the Takens-Bogdanov points are at the extremities of the Hopf-bifurcation locus described in equation (13) and of course on the Saddle-Node bifurcation locus (12). In Appendix II it is shown that the planar system undergoes a regular Takens-Bogdanov bifurcation at either of these points. The corresponding normal form is given by:

$$
\begin{aligned}
& \dot{\eta_{1}}=\eta_{2}, \\
& \dot{\eta_{2}}=\beta_{1}+\beta_{2} \eta_{1}+\eta_{1}^{2}+\eta_{1} \eta_{2} .
\end{aligned}
$$


It follows from Theorem 8.4 [13] that in the bifurcation diagram of the system (9) we encounter a line corresponding to a Hopf bifurcation creating an unstable limit cycle together with a stable focus and a homoclinic bifurcation of the saddle through which the unstable limit cycle is annihilated. The loci in the bifurcation diagram corresponding to the homoclinic bifurcations are obtained numerically and displayed in Figure 3. The Hopf-bifurcation locus is described in equation (13).

\section{Bifurcation diagram}

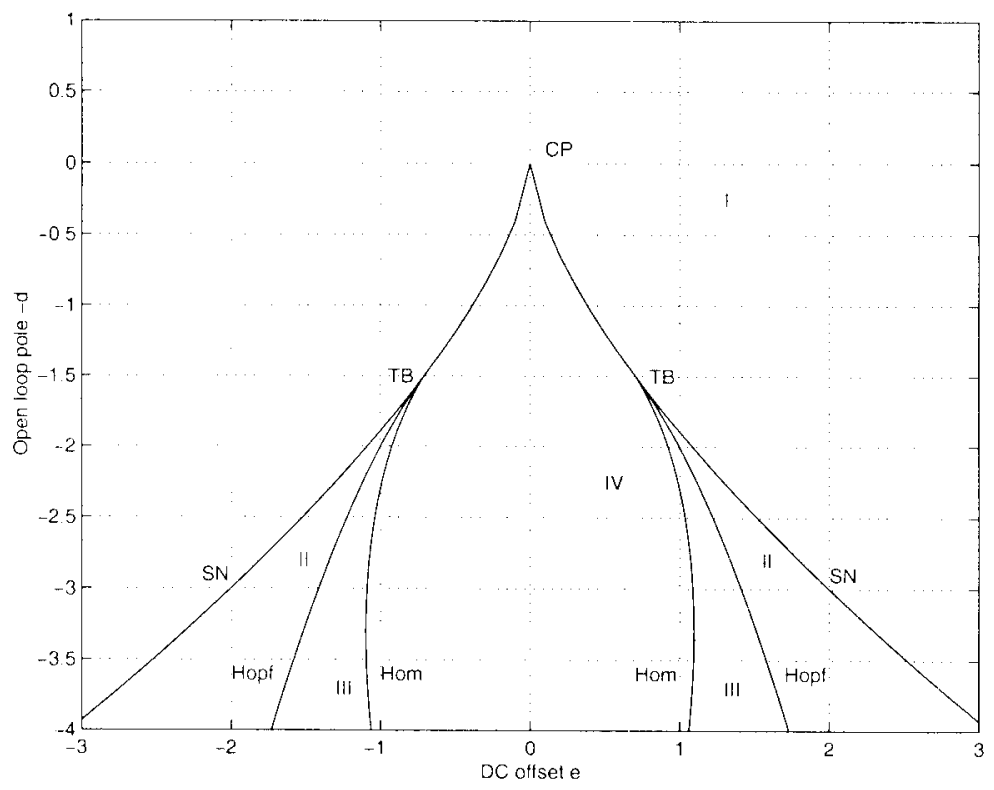

Figure 3: Bifurcation diagram for the planar adaptive system

We can now piece together a rather complete picture of the asymptotic dynamics as function of the bifurcation parameters $d$, $e$. The bifurcation diagram is depicted in Figure 3, whilst Figures 5 to 7 represent a number of typical phase portraits corresponding to the various possibilities suggested by the bifurcation diagram. In all simulations we used $\sigma=1$. The bifurcation diagram was generated using Dtool. The phase portraits were simulated using Matlab, with the aid of the suite pplane5.m.

The line SN represents the Saddle-Node bifurcation locus, equation (12). The cusp point $C P$ is the origin in the bifurcation diagram $(e, d)=$ $(0,0)$ with corresponding equilibrium $\left(y_{e}, k_{e}\right)=(0,0)$. The points $\mathbf{T B}$ identify the Takens-Bogdanov points (15) and (16), which here clearly are the points around which the bifurcation diagram can be organised. The lines Hom identify the homoclinic cycle bifurcation, terminating at 
a TB point. The lines Hopf identify the Hopf bifurcation, terminating at a TB point, see (13).

Let us follow a counterclockwise direction through the bifurcation diagram starting in the region marked $\mathbf{I}$. In region $\mathbf{I}$ there is a unique locally stable equilibrium, seemingly globally attractive (only confirmed analytically for $d>0$ ). Figure 5 depicts a representative phase portrait.

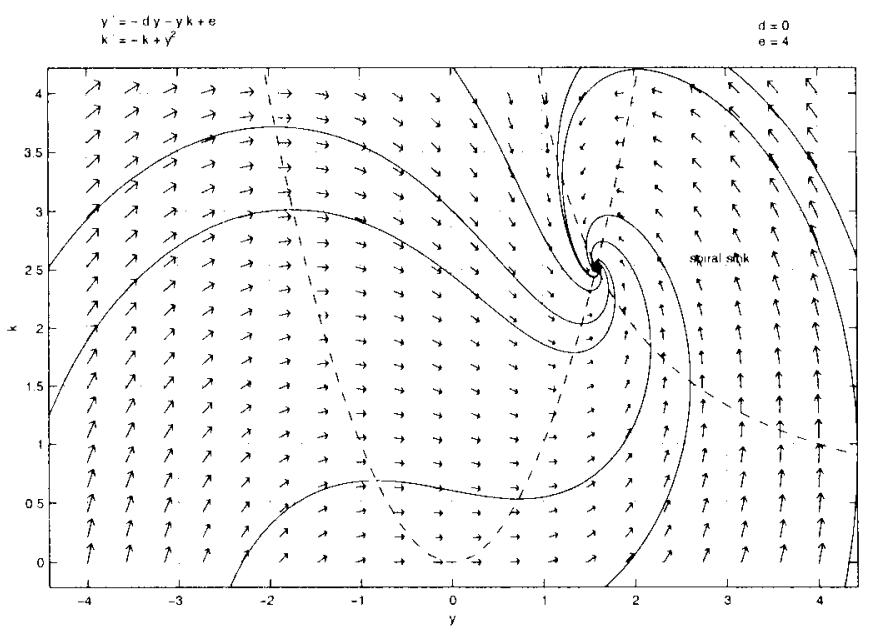

Figure 4: Phase portrait for bifurcation diagram region I in Fig. 3

In the regions II, after the saddle-node bifurcation, three equilibria coexist. A saddle and unstable focus come into existing through the SaddleNode bifurcation. It appears from the phase portrait in Figure 5 that apart from the stable manifold of the saddle, all solutions converge to the stable focus.

In the regions III, just after the Hopf bifurcation, the spiral source equilibrium sheds through the Hopf bifurcation an unstable limit cycle and becomes a stable focus. The interior of the limit cycle is the domain of attraction of this focus. The other stable equilibrium attracts the other solutions, apart from the stable manifold of the saddle. This is shown in Figure 6, which represents a typical phase portrait for Region III.

The limit cycle continues to grow (in size and period) as we further move towards the line Hom. There a homoclinic bifurcation occurs, which destroys the limit cycle. After the homoclinic bifurcation in Region IV 


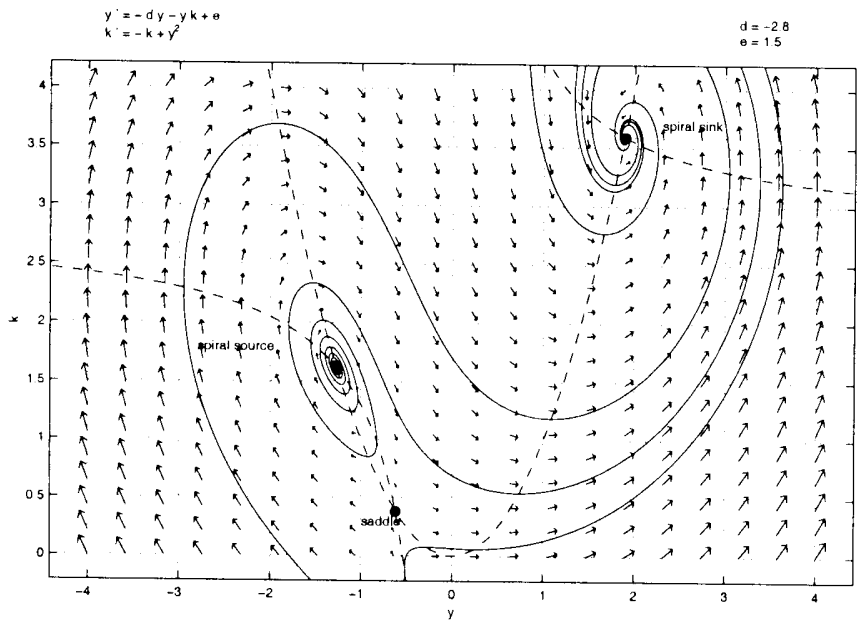

Figure 5: Phase portrait for bifurcation diagram region II in Fig 3.

it appears from Figure 7, that the stable manifold of the saddle acts as a separatrix for the domains of attraction of the stable foci. (On the line $e=0$ this was analytically demonstrated above.)

\subsection{Control interpretation}

Despite that the disturbance $e$ complicates the phase portrait in a significant way, its influence from a control perspective is not so important. Both transient behaviour (although we have not presented a complete proof for this) and asymptotic dynamics appear entirely acceptable. Certainly, the asymptotic dynamics are bounded. More importantly the output of the plant becomes of the same order of magnitude as the disturbance $e$. This is in complete accordance with linear control intuition. One simply can not expect any better from a static gain output feedback. The main difference with static output feedback is of course that through the adaptation law there are multiple coexisting possibilities for the asymptotic dynamics, but all of similar control performance.

The main drawback of the adaptive law is that there is no real control over the actually achieved control performance and that it only achieves marginal stabilization of the plant.

It could nevertheless be argued that the $\sigma$ modification with adaptive gain does work, and is robust with respect to input disturbances. This follows because over the class of systems controlled by the adaptive system it is not possible to estimate the achievable performance. Also no actual requirements 


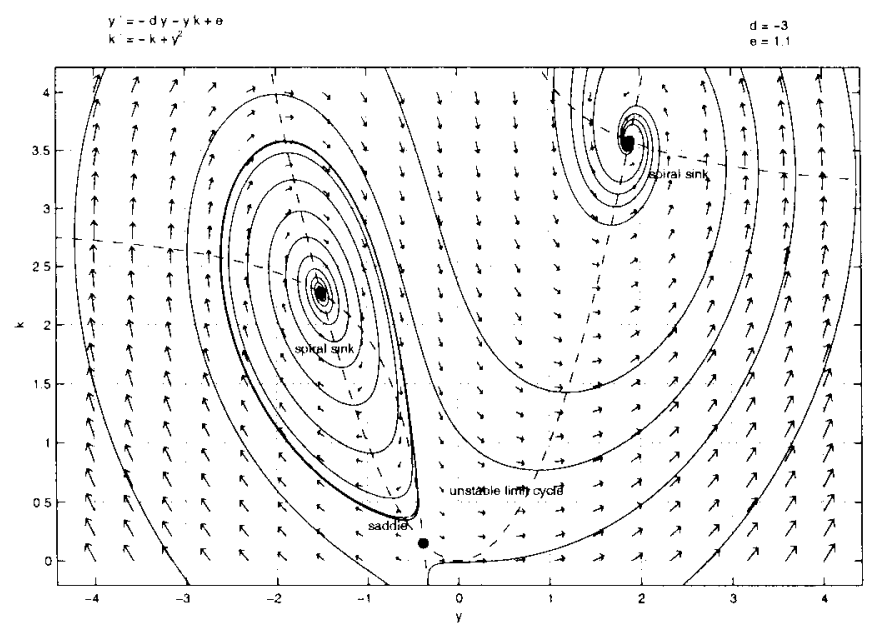

Figure 6: Phase portrait for bifurcation diagram region III in Fig 3.

are incorporated in the adaptation law, apart from the fact that we would like $y$ to be regulated. In the presence of disturbances this becomes impossible, and how much we want to reject the disturbances has not been specified at all. Hence, we could not really expect any better behaviour than what is achieved. This identifies in a sense where the suggested adaptation mechanism falls short: the achievable control performance is to be identified in order to realise a true adaptive control strategy.

The above picture is however too optimistic as will transpire from the next example.

\section{Three dimensional adaptive system}

In this section we investigate the possible asymptotic dynamics when the plant has a single stable zero, here fixed at $-a<0$. The adaptive system (1) takes the form:

$$
\begin{aligned}
\dot{x} & =-a x+y, \\
\dot{y} & =-c x-d y-k y+e, \\
\dot{k} & =-\sigma k+y^{2} .
\end{aligned}
$$

We treat the parameters $c, d$ and $e$ as bifurcation parameters. A complete bifurcation diagram will not be presented.

Before we start our discussion of the bifurcation diagram, let us observe that the phase portrait for $c=0$ is already known by virtue of our discussion in 


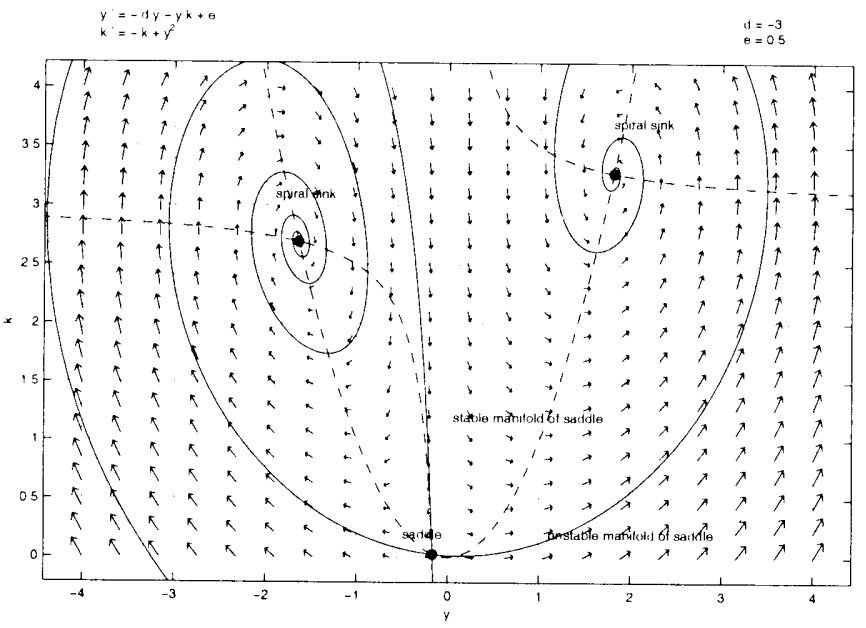

Figure 7: Phase portrait for bifurcation diagram region IV in Fig 3.

the previous section. Indeed for $c=0$ the $x$-state does not affect the $(y, k)$ dynamics, which coincide with the planar system discussed before, see equation (9). Hence the phase portrait will contain for the appropriate parameters unstable limit cycles, homoclinic orbits and multiple equilibria.

\subsection{Equilibria and Jacobian}

The equilibria are characterised by:

$$
a x_{e}=y_{e}, \quad \sigma k_{e}=y_{e}^{2}, \quad \sigma(c / a+d) y_{e}+y_{e}^{3}=\sigma e .
$$

The equilibrium is unique if

$$
c+d a \geq 0, \text { or } c+d a<0 \text { and } e^{2}>-\frac{2 \sigma}{27}(d+c / a)^{3} .
$$

There are three distinct equilibria if:

$$
c+d a<0 \text { and } e^{2}<\frac{2 \sigma}{27}(-d-c / a)^{3} .
$$

At an equilibrium the Jacobian is given by:

$$
J_{e}=\left(\begin{array}{ccc}
-a & 1 & 0 \\
-c & -d-k_{e} & -y_{e} \\
0 & 2 y_{e} & -\sigma
\end{array}\right) .
$$


The characteristic polynomial of the Jacobian, evaluated at an equilibrium, is given by;

$$
\operatorname{det}\left(\lambda I-J_{e}\right)=(\lambda+\sigma)\left(\lambda^{2}+(d+a) \lambda+c+d a\right)+k_{e}(\lambda+a)(\lambda+3 \sigma) .
$$

\subsection{Unperturbed adaptive system $e=0$}

1. An open loop stable plant $d+a>0$ and $a d+c>0$ implies that the trivial solution is globally asymptotically stable

This observation is a corollary to Theorem 3.3, as any stable second order linear system with a stable zero has indeed a strictly positive real transfer function.

2. Unstable system with unstable zero leads to unbounded response

The condition $a>0$ is necessary for stability whenever the open loop system is unstable. Consider for example the adaptive system:

$$
\begin{aligned}
\dot{x} & =-a x+y, \\
\dot{y} & =-d y-k y, \\
\dot{k} & =-k+y^{2} .
\end{aligned}
$$

Obviously, for all $a \leq 0$ we have that $x$ grows without bound (for almost all initial conditions, and all $d$ ). The adaptive feedback is only meaningful when the system has stable zeroes.

\section{Pitchfork bifurcation}

The pitchfork bifurcation locus in the bifurcation diagram is given by $c+d a=0$, see equation (21) with $e=0$. For $c+d a<0$ the origin is a saddle. The other two equilibria are described by $\left(a y_{e}, y_{e}, y_{e}^{2} / \sigma\right)$ with $y_{e}^{2}=-\sigma(c / a+d)$. Their local stability is determined from the location of the roots of (23):

$$
\lambda^{3}+\frac{1}{a}\left(a \sigma+a^{2}-c\right) \lambda^{2}+\frac{\sigma}{a}\left(-2(a d+c)-c+a^{2}\right) \lambda-2 \sigma(a d+c)
$$

These cquilibria are stable when $a^{2}-c>\max (a \sigma,-2(a d+c))$, and $\left(a^{2}-c\right)\left(a^{2}+a \sigma-c\right)-2(a d+c)(a \sigma-c)>0$ unstable otherwise. This means that immediately after the pitchfork bifurcation, i.e. for $a d+c<0$ but close to 0 , the new equilibria are locally stable if $d+a>0$, and saddles otherwise.

4. Takens-Bogdanov point: open loop system is double integrator The origin is a Takens-Bogdanov point when $d+a=0$ and $a d+c=0$. The corresponding Jacobian has two zero eigenvalues with geometric multiplicity one, and one eigenvalue $-\sigma$. Using the comparison function 
$V=x^{2}+y^{2}+k^{2}-4 k$, it can be verified that the origin is globally asymptotically stable in this situation.

The bifurcation locus for the homoclinic orbit bifurcation is contained in the parameter domain $0<c<a^{2}$ and $d<-a$ (see next item Hopf bifurcation).

\section{Hopf bifurcation}

The origin undergoes a regular Hopf bifurcation for $d+a=0$ and $a d+c>0$. The origin remains the unique equilibrium, but becomes an unstable focus as $d+a$ becomes negative. A stable periodic orbit comes into existence which persists for all $d<-a$ and $c>a^{2}$, at least for sufficiently small $\sigma$. This can be demonstrated using averaging ideas. The calculations are summarized in Appendix III and IV. In Appendix III we establish a domain of attraction for an invariant torus, which we demonstrate to contain a unique periodic orbit in Appendix IV. Appendix IV provides existence, uniqueness and local stability. It is demonstrated that the periodic orbit is for sufficiently small $\sigma$ well approximated by:

$$
\begin{aligned}
x(t, \sigma) & =-\sqrt{\frac{-2 \sigma(d+a)}{1+a^{2}}} \sin (\omega t)+O(\sigma), \\
y(t, \sigma) & =\sqrt{\frac{-2 \sigma(d+a)}{1+a^{2}}}(-a \sin (\omega t)+\omega \cos (\omega t))+O(\sigma), \\
k(t, \sigma) & =-(d+a)+O(\sqrt{\sigma}), \\
\omega^{2} & =c-a^{2} .
\end{aligned}
$$

In Appendix III it is demonstrated that the domain of attraction is virtually all of $\mathbb{R}^{3}$ apart from the axis $\{x=y=0\}$ (more precisely, any bounded domain in $\mathbb{R}^{3} /\{x=y=0\}$ is contained in a domain of attraction for sufficiently small $\sigma$ ).

The above observations complete the local bifurcation picture for the trivial equilibrium.

Following the non-zero equilibria it can be observed that they too undergo a Hopf bifurcation, creating locally attractive limit cycles. This takes place for $a d+c<0$ and $\left(\sigma a+a^{2}-c\right)\left(a^{2}-c-2(a d+c)\right)=-a^{2}(a d+c)$. The limit cycles themselves undergo a sequence of saddle-node bifurcations, eventually evolving into complex attractors. These limit cycles and attractors do not scale with $\sigma$ as the limit cycles originating from the Hopf bifurcating origin (26). An example is represented in the Figure 8. The aperiodic response in Figure 8 is generated from the initial condition $\left(x_{0}, y_{0}, k_{0}\right)=(0,1,1)$ for the system (18) with $a=1, d=-2.751, c=-5.301, \sigma=0.001$. It is reminiscent of drift-burst behaviour observed in other direct adaptive control schemes [2]. A partial, numerically obtained bifurcation diagram is represented in Figure 9. The bifurcation parameter is $\alpha$. The system (18) has parameters $a=1, d=$ 


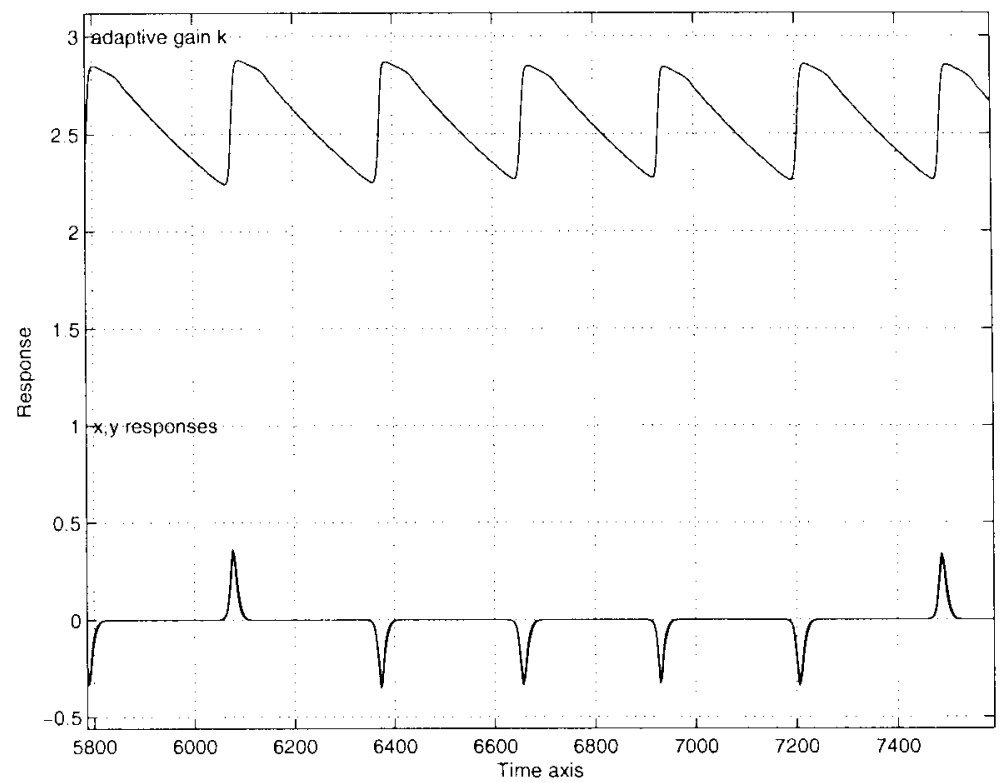

Figure 8: Aperiodic behaviour after pitch fork bifurcation followed by Hopf bifurcations

$-\alpha, c=-\alpha / 4+3 / 4$ and $\sigma=-0.1$. The bifurcation parameter ranges from 0 to 2.5. A phase space portrait displaying some of the possible competing asymptotic dynamics for $\alpha=0.82$ is displayed in Figure 10. The numerical bifurcation diagram reveals that the origin undergoes a pitch fork bifurcation $\alpha=0.6$. The origin remains unstable for all $\alpha>0.6$. The new equilibria are unstable in the interval $0.831<\alpha<1.86$, and locally asymptotically stable otherwise. In the same parameter region $0.831<\alpha<1.86$ we identify a strange attractor, with a structure not dissimilar from the Lorenz attractor. The non zero equilibria undergo regular Hopf bifurcations for $\alpha=0.831$ and 1.86. The Hopf bifurcations are supercritical. Regions of hysteresis are clearly identifiable, indicative of saddle node bifurcations for the limit cycles.

\subsection{Perturbed adaptive system}

We now consider concisely the influence of the input offset $e$.

1. Stable plant: $c+a d>0$ and $d+a>0$

Under these condition there is a unique equilibrium, sce cquation (19), which is at least locally uniformly stable, see equation (23). The output is no longer regulated, at equilibrium $\left|y_{e}\right|<|e|$. 


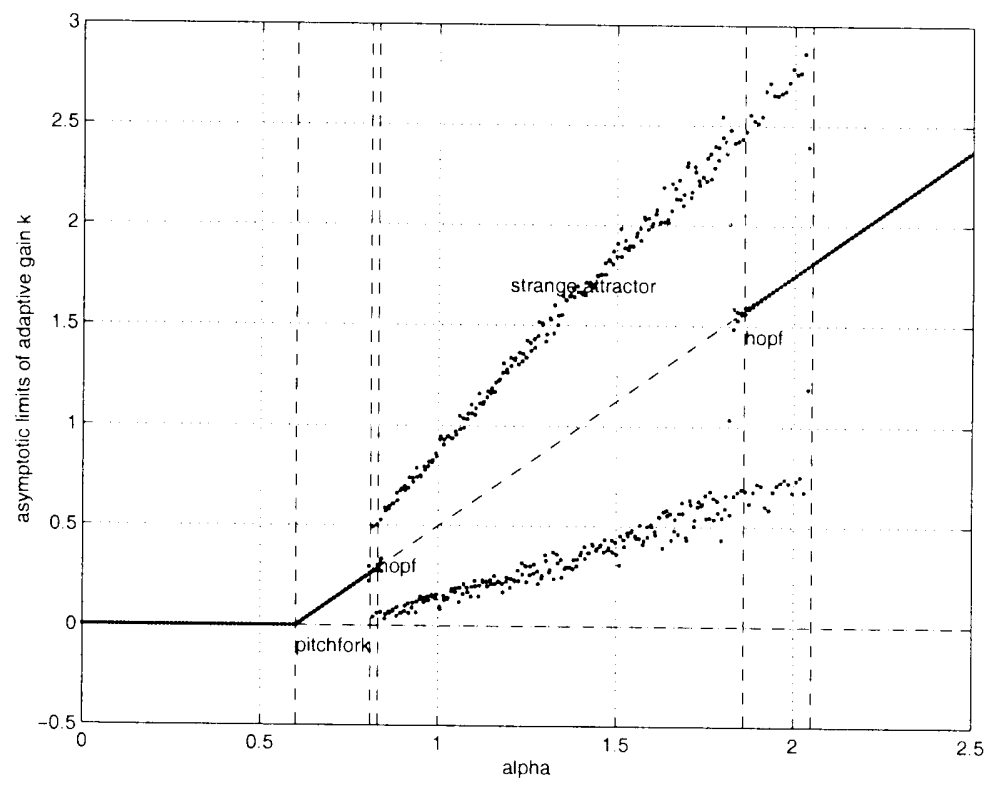

Figure 9: Partial bifurcation diagram for system (18)

2. Unstable plant, real poles: $c+d a<0$

In this situation there may be up to three equilibria, (see equation (19), depending on the magnitude of $e$. The main event in this part of the bifurcation diagram is the saddle-node bifurcation. This situation corresponds very much to the planar system discussed above. It suffices to consider the local dynamics in the centre manifold after eliminating a one dimensional stable manifold.

(a) The saddle-node bifurcation locus is given by

$$
e^{2}=\frac{2 \sigma}{27}(-d-c / a)^{3}
$$

For $e^{2}>\frac{2 \sigma}{27}(-d-c / a)^{3}$ there is but one locally stable equilibrium, for $e^{2} \leq \frac{2 \sigma}{27}(-d-c / a)^{3}$ there are three equilibria.

The control interpretation is that a larger DC offset enforces a larger control gain, which in turn provides more stability. 


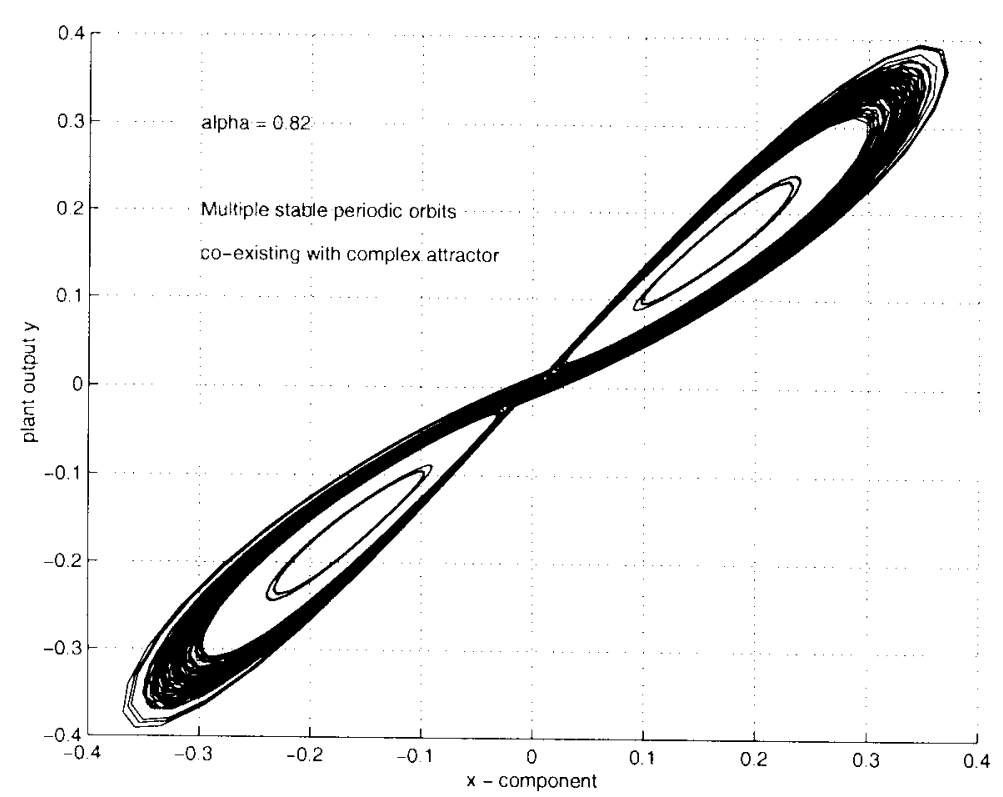

Figure 10: Co-existing asymptotically stable dynamics for (18)

(b) Takens-Bogdanov points There is a variety of Takens-Bogdanov points characterised by:

$$
\begin{aligned}
\left(\sigma+a-\sigma a-1 / 3 a^{2}\right) d+(1+\sigma-a / 3) c & =-\sigma a, \\
-4 \sigma(c+a d)^{3}+27 e^{2} & =0, \\
k_{T B} & =-\frac{c+a d}{3}, \\
y_{T B}^{2} & =\sigma k_{T B}, \\
a x_{T B} & =y_{T B} .
\end{aligned}
$$

(c) Organising centres

The most degenerate bifurcation points, also the most likely organising centres in the bifurcation diagram are those equilibria with a zero eigenvalue of algebraic multiplicity three and with a single eigenvector. One expects to find complex asymptotic attractors in the neigbourhood of such points. These points only exist if 
$\sigma>a>0$, and are characterised by:

$$
\begin{aligned}
d & =\frac{2 a^{2}-3 \sigma^{2}}{2(\sigma-a)}, \\
c & =\frac{a^{3}}{a-\sigma)} \\
e^{2} & =\frac{2 \sigma^{7}}{(\sigma-a)^{3}}, \\
k_{e} & =\frac{\sigma^{2}}{2(\sigma-a)}, \\
y_{e}^{2} & =\frac{\sigma^{3}}{2(\sigma-a)}, \\
a x_{e} & =y_{e} .
\end{aligned}
$$

These points, because they only exist when $\sigma>a$, are less relevant from a control perspective as one typically selects $\sigma$ to be small. Nevertheless, this information serves to qualify what we have to understand under small $\sigma$, and furthermore serves to underscore the difficulty in selecting an appropriate $\sigma$ value. Indeed, a proper value of $\sigma$, if one wants to avoid the complex dynamics associated with such points, does require non trivial prior knowledge about the location of the plant transfer function zeroes.

\section{Hopf bifurcations}

In case $c+a d>0$ there is unique equilibrium, that is locally asymptotically stable for $d+a>0$, and may undergo a Hopf bifurcation for $d+a \leq 0$. The Hopf bifurcation locus corresponding to this bifurcation situation is described by:

$$
\begin{aligned}
a^{2} e^{2} & =\sigma k(c+d+k)^{2}, \\
(c+a d)+\sigma(a+d)+(a+3 \sigma) k & >0, \\
(\sigma+d+a+k)(a \sigma+c+(a+\sigma) d+k(a+3 \sigma)) & =\sigma((c+a d)+3 a k) .
\end{aligned}
$$

(The elimination of $k$ from the above expression (30)) is feasible, but leads to a rather cumbersome and uninteresting semi-algebraic expression.) Given that the constant term in (23) is always positive, we observe that a stable focus looses stability and a locally stable limit cycle is created at the Hopf bifurcation. In case $e=0$, the origin is the only equilibrium, and the Hopf bifurcation takes places at $d=-a$. The averaging results established in Appendix III and IV can be repeated for this situation as well.

The equilibria generated through the saddle node bifurcation also undergo a Hopf bifurcation. 


\subsection{Control interpretation}

The adaptive control law fails to achieve regulation in all but the rather noninteresting case of a stable open loop system. The presence of a DC offset has two main effects, first it acts stabilising in that it enforces a larger feedback gain than would have been the case without it, but it also induces further complex dynamics in the behaviour depending on the actual plant parameters. We conclude that the $\sigma$ modification limits the adaptive gain too severely to achieve the desired control objective. Moreover we observe that the selection of $\sigma$ is not trivial. A proper selection, one that avoids as much as feasible complicated dynamics and thus assists in predicting the typical asymptotic dynamics, requires a $\sigma$ value which is small compared to the dominant time constants and zeroes of the plant's transfer function.

\section{Generalisations}

In higher dimensions, the system (3) with $n>1$, all the above discussed bifurcation phenomena re-occur and more complicated ones emerge.

We limit the discussion to the generic bifurcations corresponding to equilibria. The saddle-node bifurcation phenomenon we observed in the planar system and the Hopf bifurcation we observed in the three dimensional example are the main events to consider.

The emphasis is on a control theoretic interpretation.

\subsection{Equilibria}

For the general case, see equation (3), the equilibria $\left(x_{e}, y_{e}, k_{e}\right)$ satisfy the equations:

$$
\begin{aligned}
x_{e} & =-A^{-1} b y_{e}, \\
k_{e} & =\frac{y_{e}^{2}}{\sigma}, \\
\sigma e & =\sigma\left(-c A^{-1} b+d\right) y_{e}+y_{e}^{3} .
\end{aligned}
$$

Provided $d-c A^{-1} b=p(0)+d z(0) \geq 0$ there is but one equilibrium. Notice also that in this case $\left|y_{e}\right| \leq|e|$ and $y_{e}$ has the same sign as $e$.

If $d-c A^{-1} b<0$ there are possibly three equilibria, depending on the magnitude of $e$. At least one of the equilibria is larger in magnitude than

$$
y^{*}=\sqrt{-\sigma\left(d-c A^{-1} b\right)} ; \text { whenever } d-c A^{-1} b<0 .
$$

For future reference we denote

$$
k^{*}=y^{* 2} / \sigma=c A^{-1} b-d .
$$


There are three equilibria only if

$$
|e| \leq e^{*}=\frac{2\left(c A^{-1} b-d\right)^{3 / 2}}{3 \sqrt{3}}
$$

The equation (34) represents the saddle-node bifurcation locus in the bifurcation diagram. When the condition (34) is satisfied, for one equilibrium the output value is larger in magnitude than $y^{*}$, this equilibrium we refer to as $y_{e 1}$. Another equilibrium has an output value contained in the interval $-y^{*} / \sqrt{3} \leq y_{e 2} \leq y^{*} / \sqrt{3}$. The last equilibrium is such that the output value has the opposite sign of $y_{e 1}$ and satisfies $y^{* 2} \geq y_{e 3} \geq y^{* 2} / 3$.

\subsection{Local stability of equilibria}

The characteristic equation of the Jacobian evaluated at the equilibria is given by:

$$
\begin{aligned}
J(\lambda) & =\operatorname{det}\left(\begin{array}{ccc}
\lambda I-A & -b & 0 \\
c & \lambda+d+k_{e} & y_{e} \\
0 & -2 y_{e} & \lambda+\sigma
\end{array}\right), \\
& =(\lambda+\sigma)\left(p(\lambda)+\left(d+k_{e}\right) z(\lambda)\right)+2 y_{e}^{2} z(\lambda) \\
& =(\lambda+\sigma)(p(\lambda)+d z(\lambda))+k_{e}(\lambda+3 \sigma) z(\lambda) .
\end{aligned}
$$

From this it follows that for sufficiently small $\sigma$ the root loci of the plant $p(\lambda)+\left(d+k_{e}\right) z(\lambda)$ as a function of $k_{e}$ is a good indicator for what to expect in the phase portrait of the adaptive system. (Again sufficiently small $\sigma$ means small with comparison to the dominant time constants and zeroes of the plant's transfer function.)

\subsubsection{Case 1: Open loop stable plant}

The origin is a locally asymptotically stable equilibrium in this case (see equation (35) with $k_{e}=0$. If moreover the plant has a strictly positive real transfer function, then the equilibrium is globally asymptotically stable. The local analysis conforms of course. Indecd, in this case $p(\lambda)+(d+k) z(\lambda)$ is a Hurwitz polynomial for all $k \geq 0$. Hence $p(0)+d z(0)>0$ which confirms that the equilibrium is unique and from equation (35) we observe that it is locally stable.

In general, for stable and minimum phase but not strictly positive real plants, there will be multiple asymptotically stable attractors in the phase portrait, the origin being one of them. See the example (Figure 1) at the end of Section 3 for an illustration. (It can be verified that the domain of attraction of the origin is rather small.) 


\subsubsection{Case 2: Open loop unstable plant, single positive pole}

In this scenario, the open loop plant is unstable with a single positive eigenvalue; the root loci of $p(\lambda)+(d+k) z(\lambda)$ with $k$ viewed as a parameter, has a single (real axis) branch cutting the imaginary axis for $k=k^{*}=-p(0)-d z(0)$. In this case we have obviously $p(0)+d z(0)<0$. There may be as many as three equilibria, see equation (34). The equilibrium corresponding to $y_{e 1}$ for which $\left|y_{e 1}\right| \geq y^{*}$ is also locally asymptotically stable. Indeed we can rewrite the Jacobian (35) in this case as: $J(\lambda)=(\lambda+\sigma)\left(p(\lambda)+\left(d+k_{e}\right) z(\lambda)\right)+2 y_{e}^{2} z(\lambda)$. Because $p(\lambda)+\left(d+k^{*}\right) z(\lambda)=\lambda h(\lambda)$ for some Hurwitz polynomial $h(\lambda)$. It follows that under this scenario with $k_{e} \geq k^{*}, J(\lambda)$ is Hurwitz.

The cquilibrium with $y_{e 2}$ is always unstable, because $J(0)=\sigma(p(0)+d z(0))+$ $3 y_{e 2}^{2} z(0) \leq 0$.

The equilibrium corresponding to $y_{e 3}$ may be either stable or unstable.

Clearly the transition from Case 1 to Case 2 is effectuated via a saddlenode bifurcation, e.g. associated with a variation of the parameter $d$. The bifurcation value being $d=d_{p}$ for which $p(0)+d_{p} z(0)=0$. This phenomenon is completely captured by the planar example.

But as indicated in the three dimensional example, depending on the plant, the global dynamics may be rather more complicated with possibly co-existing attractors, cither multiple locally stable equilibria, but possibly also periodic orbits and even complex attractors, see Figure 10.

\subsubsection{Case 3: a single pair of complex conjugate unstable poles}

When the open loop plant is unstable with a single pair of complex conjugate cigenvalues, we have that $p(0)+d z(0)>0$, and there is but one equilibrium. The local stability now depends in a crucial manner on the magnitude of the disturbance $e$. The transition from Case 1 to Case 3 corresponds to a Hopf bifurcation.

In particular for $e=0$, the equilibrium is the origin $\left(x_{e}, y_{e}, k_{e}\right)=(0,0,0)$ and clearly this equilibrium is unstable, the Jacobian bcing equal to $J(\lambda)=$ $(\lambda+\sigma)(p(\lambda)+d z(\lambda))$, which by assumption has a single pair of complex conjugate roots with positive real part. In this situation the adaptive system adjust $k$ such that a stable limit cycle comes into existence. Using averaging ideas as in the Appendix IV we may show that the limit cycle, for sufficiently small $\sigma$, is well approximated by:

$$
\begin{aligned}
& x(t)=O(\sqrt{\sigma}), \\
& y(t)=\sqrt{2 \sigma k_{0}} \sin (\omega t+\phi)+O(\sigma), \\
& k(t)=k_{0}+O(\sqrt{\sigma}) .
\end{aligned}
$$

Where $k_{0}$ and $\omega$ are such that:

$$
\operatorname{det}\left(j \omega I-\left(\begin{array}{cc}
-A & -b \\
c & d+k_{0}
\end{array}\right)\right)=0 .
$$


As $y_{e}$ and hence the corresponding $k_{e}$ increases monotonically with $e$ it follows that under this scenario at some critical level the equilibrium undergoes a Hopf bifurcation. For $|e|>e_{H}$ ( such that $k_{e}=k_{0}$ ) the equilibrium is locally asymptotically stable, for $|e| \leq e_{H}$ it is unstable and a stable limit cycle exists. Again it is a direct consequence of the center manifold theorem that the three dimensional example captures the essence of the local dynamics. There exists an asymptotically stable periodic orbit for all $|e|<e_{H}$ and the equilibrium is asymptotically stable otherwise.

\subsection{Other scenarios}

The transition from a stable plant to an unstable plant via a Saddle-Node or Hopf bifurcation is but the simplest scenario. Obviously, much more complicated bifurcation phenomena are possible. Nevertheless, within the class of linear systems considered, these are the generic bifurcations. The different asymptotic dynamics corresponding to these transitions being explored in the above two and three dimensional examples.

Typically the least structurally stable (and hence most interesting) plants to consider are those linear plants that have all their poles in the origin. In the bifurcation diagram for such adaptive systems (order $>2$ ) we expect to encounter chaotic dynamics.

Moreover as for a typical plant, there may be multiple values of the feedback gain $k$ for which the plant is marginally stabilised, there will be in the phase portrait of the adaptively controlled system a multiplicity of different and co-existing asymptotic dynamics, each corresponding to a different level of adaptive feedback gain.

\section{Alternative adaptive gain laws}

Clearly the adaptive systems we discussed may exhibit a rather rich variety of asymptotic dynamics. One may wonder, could we design an adaptive gain controller of the form $\dot{k}=f(k, y)$ with smooth $f$ that achieves regulation in a structurally stable manner, i.e. without displaying periodic behaviour and or multiple co-existing asymptotic dynamics for all sufficiently small perturbations of the vector field. (Allowing for perturbations other than the constant input offset considered here.) Unless we are given more prior information about the class of systems which need to be controlled, the answer appears to be negative.

Indeed, the class of systems to be controlled, linear systems with a transfer function characterised by stable zeroes and pole-zero excess of one, is simply too rich. Regardless of the adaptive gain law $f(k, y)$ we use to control the linear system at hand, the closed loop dynamics may exhibit the richness we discussed above.

In order to find a structurally stable adaptive gain law we need to consider adaptation laws of the form $\dot{z}=f(z, y)$ with $k=g(z, y)$ or perhaps $\dot{k}=$ 
$f(k, y, t)$. These options are presently under consideration.

Alternatively, we may want to invoke further prior information on the systems to be controlled in order to achieve robust adaptive regulation.

Let us observe that the bifurcation analysis has provided us with a lot of information about the behaviour of these systems, and this knowledge can be exploited to design a supervisory adaptive control law to decide on an appropriate feedback gain level. A supervisor could observe the asymptotic dynamics, from a catalog decide in which regime the adaptive system is operating and reset the feedback gain accordingly. An adaptive feedback law building on this principle could take on the form:

$$
\dot{k}=-\sigma\left(k-k_{r}\right)+y^{2} .
$$

Where both the value of $\sigma$ and $k_{r}>0$ are set by the supervisor. Appropriate selection rules for these parameters are under investigation.

\section{Conclusions}

Through a bifurcation analysis, we explored the possible asymptotic dynamics in adaptive high gain feedback applied to linear almost passive systems, when the adaptive law has been modified with the so called $\sigma$-modification, $\dot{k}=$ $-\sigma k+y^{2}$. In the disturbance free case, when the uncontrolled plant does not possess a strictly positive real transfer function, even when the plant is stable and minimum phase, asymptotically periodic and complex dynamics are to be expected. For the less interesting case of plants with strict positive real transfer function the system is regulated.

It became apparent that even in this simple situation the design of the adaptive law, the selection of $\sigma$ is not trivial. A proper selection requiring some information about the size of the time constants and zeroes of the plant's transfer function.

Despite the fact that the adaptive control law always leads to a bounded system response regardless of the plant, the main alarming observation is that the adaptation leads at best to a control gain which only achieves marginal stability. Hence the adaptation law can never be switched off, or stability may be lost. We conjecture that no smooth time-invariant adaptive gain law of the form $\dot{k}=f(k, y)$ can be constructed which possesses the property of regulation for the class of linear systems with stable zeroes and pole-zero excess of one, in a structurally stable manner.

The results here should be seen as a first step in the re-exploration of the adaptive control question with the aim of understanding the dynamics of adaptive systems as to develop a supervisory control approach.

Finally let us observe that we have not really dealt with the issue of transient performance in gain adaptive systems. 


\section{System representation}

For the sake of completeness we explain here how one can arrive at the (1) representation for systems that possess a transfer function of relative degree one and with stable zerocs.

Let us start from the input/output description of the system:

$$
P\left(\frac{d}{d t}\right) y=Q\left(\frac{d}{d t}\right) u
$$

where $P(\xi)$ is a monic polynomial of degree $n+1$ and $Q(\xi)$ is a polynomial of degree $n$. We assume that the system is controllable. This corresponds to $P(\xi)$ and $Q(\xi)$ being coprime. Since the degree of $P(\xi)$ is exactly one larger than the degree of $Q(\xi)$, we can write $P(\xi)=\frac{1}{q_{n}}(\xi-d) Q(\xi)+r(\xi)$ with $\operatorname{deg} r(\xi)<\operatorname{deg} Q(\xi)$. Equation (38) can then be rewritten as:

$$
\left(\frac{1}{q_{n}}\left(\frac{d}{d t}+d\right)+r\left(\frac{d}{d t}\right)\right) y=Q\left(\frac{d}{d t}\right) u
$$

Using elimination theory, [1, Chapter 6$]$ it can be secn that (39) is input/output equivalent to

$$
\frac{d}{d t} y=-d y+q_{n} u_{1}, \quad Q\left(\frac{d}{d t}\right) u_{2}=r\left(\frac{d}{d t}\right) y, \quad u=u_{1}+u_{2} .
$$

Notice that since $\operatorname{deg} Q(\xi)>\operatorname{deg} r(\xi)$, the second equation in (40) defines a proper input/output relation between $y$ and $u_{2}$. Notice also that since $P(\xi)$ and $Q(\xi)$ are coprime, so are $Q(\xi)$ and $r(\xi)$. Therefore the $\left(y, u_{2}\right)$ system is controllable. We can therefore consider the controller canonical state space representation of that system, which is given by

$$
\frac{d}{d t} x=A x+b y \quad u_{2}=h x,
$$

where the eigenvalues of $A$ are the zeroes of $Q(\xi)$ and $(A, b)$ is controller canonical form. Combining (41) with the first and the third equation of (40), we obtain

$$
\begin{aligned}
& \frac{d}{d t} x=A x+b u, . \\
& \frac{d}{d t} y=-q_{n} h x-d y+q_{n} u .
\end{aligned}
$$

Putting $c=q_{n} h$ and $g=q_{n}$ yields (1).

\section{Normal form representation for the Takens- Bogdanov point in (9)}

Without loss of generality we start with the system description (9) with $\sigma=1$, see Fact (vi). The Takens-Bogdanov points are equilibria with a nil potent Jacobian. 
For the system $(9)$ (with $\sigma=1$ ) these are given by:

$$
y_{T B}=\frac{1}{\sqrt{2}}, \quad k_{T B}=\frac{1}{2}, \quad d_{T B}=-\frac{3}{2}, \quad e_{T B}=-\frac{1}{\sqrt{2}},
$$

and

$$
y_{T B}=-\frac{1}{\sqrt{2}}, \quad k_{T B}=\frac{1}{2}, \quad d_{T B}=-\frac{3}{2}, \quad e_{T B}=\frac{1}{\sqrt{2}} .
$$

We consider the local behaviour around the latter. The former, due to symmetry will have mutatis mutandis a completely similar normal form. Introduce new variables $\left(x_{1}, x_{2}, \lambda_{1}, \lambda_{2}\right)$ through:

$$
\begin{aligned}
y & =-\frac{1}{\sqrt{2}}+x_{1}, \\
k & =\frac{1}{2}+x_{2}, \\
d & =-\frac{3}{2}-\lambda_{1}, \\
e & =\frac{1}{\sqrt{2}}+\lambda_{2}+\frac{1}{\sqrt{2}} \lambda_{1} .
\end{aligned}
$$

The system (9) described in the new variables takes on the form:

$$
\begin{aligned}
& \dot{x_{1}}=\lambda_{2}+\left(1+\lambda_{1}\right) x_{1}+\frac{1}{\sqrt{2}} x_{2}-x_{2} x_{1}, \\
& \dot{x_{2}}=-\sqrt{2} x_{1}-x_{2}+x_{1}^{2} .
\end{aligned}
$$

We now transform the variables such as to reduce the first equation to a pure integrator. (This also ensures that the lincar part for $\left(\lambda_{1}, \lambda_{2}\right)=(0,0)$ is in Jordan form.) We use the transformation:

$$
\begin{aligned}
& y_{1}=x_{1}, \\
& y_{2}=\lambda_{2}+\left(1+\lambda_{1}\right) x_{1}+\frac{1}{\sqrt{2}} x_{2}-x_{2} x_{1} .
\end{aligned}
$$

This transformation is invertible in a neighbourhood of the origin contained in the domain $y_{1}<\frac{1}{\sqrt{2}}$. The invorse is given by:

$$
\begin{aligned}
& x_{1}=y_{1} \\
& x_{2}=\frac{-\lambda_{2}+y_{2}-\left(1+\lambda_{1}\right) y_{1}}{\frac{1}{\sqrt{2}}-y_{1}} .
\end{aligned}
$$

This leads to a system description of the form:

$$
\begin{aligned}
& \dot{y_{1}}=y_{2} \\
& \dot{y_{2}}=\lambda_{2}+\lambda_{1} y_{1}+\lambda_{1} y_{2}+\frac{3}{\sqrt{2}} y_{1}^{2}-y_{1}^{3}+\sqrt{2} y_{2} \frac{\lambda_{2}-\left(1+\lambda_{1}\right) y_{1}-y_{2}}{1-\sqrt{2} y_{1}} .
\end{aligned}
$$


Only retaining terms up to second order in $\left(y_{1}, y_{2}\right)$ we arrive at a locally equivalent vector field:

$$
\begin{aligned}
\dot{z_{1}}= & z_{2}, \\
\dot{z_{2}}= & \lambda_{2}+\lambda_{1} z_{1}+\left(\lambda_{1}+\sqrt{2} \lambda_{2}\right) z_{2}-\frac{3}{\sqrt{2}} z_{1}^{2}+ \\
& \quad\left(2 \lambda_{2}-\sqrt{2}\left(1+\lambda_{1}\right)\right) z_{1} z_{2}-\sqrt{2} z_{2}^{2} .
\end{aligned}
$$

Following Section 8.4.1 in [13], the term linear in $z_{2}$ in the $z_{2}$-equation can be eliminated by a parameter dependent shift of $z_{1}$. The $z_{2}^{2}$ term in the $z_{2}$ equation can be eliminated through a time scaling transformation. Finally obscrve that the $z_{1} z_{2}$ term and the $z_{1}^{2}$ term have both negative sign, which can be made positive through the linear transformation $\left(z_{1}, z_{2}\right) \rightarrow\left(-z_{1},-z_{2}\right)$. It follows that the normal form for the Takens-Bogdanov bifurcation in the system (9) is given by:

$$
\begin{aligned}
& \dot{\eta_{1}}=\eta_{2}, \\
& \dot{\eta_{2}}=\beta_{1}+\beta_{2} \eta_{1}+\eta_{1}^{2}+\eta_{1} \eta_{2} .
\end{aligned}
$$

\section{Averaging computations}

Consider the system (18) and assume that $\sigma<<1$. Given Fact (vi), this is actually without much loss of generality, as we can always scale the vector ficld as to satisfy this condition.

Consider the following time varying state space transformation:

$$
\begin{aligned}
R & =\left(\begin{array}{cc}
-a & 1 \\
-c & a
\end{array}\right) \\
z & =\frac{1}{\sqrt{\sigma}} \exp (-R t)\left(\begin{array}{l}
x \\
y
\end{array}\right) \\
\xi & =\frac{k+a+d}{\sqrt{\sigma}} .
\end{aligned}
$$

Notice that $\exp (R t)$ is a rotation matrix. Let $\omega=\sqrt{c-a^{2}}$. Let $b^{T}=\left(\begin{array}{ll}0 & 1\end{array}\right)$. We have that $(z, \xi)$ satisfy the following differential equation:

$$
\begin{aligned}
& \dot{z}=\sqrt{\sigma} \xi \exp (-R t) b b^{T} \exp (R t) z, \\
& \dot{\xi}=-\sigma \xi-\sqrt{\sigma}\left(-a-d-z^{T} \exp \left(R^{T} t\right) b^{T} b \exp (R t) z\right) .
\end{aligned}
$$

This is in standard form to apply averaging techniques ([9]), using $\sqrt{\sigma}$ as a small parameter. The averaged system of equations is given by:

$$
\begin{aligned}
\dot{z}^{a} & =\sqrt{\sigma} \xi^{a} \Gamma z^{a}, \\
\dot{\xi^{a}} & =-\sigma \xi^{a}-\sqrt{\sigma}\left(-a-d-z^{a T} \Delta z^{a}\right) .
\end{aligned}
$$


For a first order averaging approximation one could neglect the term $-\sigma \xi^{a}$. Nevertheless, because we are interested in an approximation valid over the entire time axis, and because this term provides stability, we carry it along in the averaged equation. For a discussion of the validity of this approach see c.g.[9] Chapter 4.

Here $\Gamma$ and $\Delta$ are given by:

$$
\Gamma=\frac{1}{2 \omega^{2}}\left(\begin{array}{cc}
\omega^{2}+1 & -2 \\
c & \omega^{2}-1
\end{array}\right) \quad \Delta=\frac{1}{2 \omega^{2}}\left(\begin{array}{cc}
c^{2} & -c \\
-c & \omega^{2}+1
\end{array}\right)
$$

Importantly observe that $\Delta \Gamma+\Gamma^{T} \Delta=\Delta=\Delta^{T}>0$.

Let us consider the following comparison function for the averaged system (54).

$$
V\left(z^{a}, \xi^{a}\right)=\frac{1}{2}\left(z^{a^{T}} \Delta z^{a}+d+a\right)-\frac{\sqrt{\sigma}}{2} \log \frac{z^{a T} \Delta z^{a}}{-a-d}+\frac{1}{2} \xi^{a^{2}} .
$$

Notice that $V$ is positive on the domain $D=\left\{\left(z^{a}, \xi^{a}\right): z^{a} \neq 0\right\}$, and achicves a minimum $V=0$ on the ellipse $E=\left\{\left(z^{a}, \xi^{a}\right): \xi^{a}=0 ; z^{a T} \Delta z^{a}=-a-\right.$ $d\}$. Moreover $V$ grows unbounded whenever its argument approaches the boundary of the domain $D$.

Assuming that $z^{a}(0) \neq 0$, we have that along the solutions of the avcraged system equations (54) $V\left(z^{a}(t), \xi^{a}(t)\right)=-\sigma \xi^{a}(t)^{2} \leq 0$. Using LaSalle's invariance principle we deduce that all solutions starting inside $D$ converge to a fixed point in $E$. Locally this convergence is exponentially fast.

From the results in Chapter 4 [9] it follows that the original system of equations (53) contains an attractive invariant tubular neighbourhood of radius $O(\sqrt{\sigma})$ centred on the ellipse $E$ with a domain of attraction arbitrarily large inside the interior of $D$. The convergence is exponential. (Considering a larger domain of attraction, within $D$, may require one to consider smaller values of $\sigma$.

Re-interpreting the result in terms of the original coordinates (18) we obtain:

Theorem III.1 Consider the system (18) under the conditions $e=0, d+a<$ 0 and $c+a d>0$. Let $x(0) \neq 0$ and $k(0) \geq 0$. For sufficiently small $\sigma>0$, all solutions satisfy:

$$
\begin{aligned}
& \limsup _{t \rightarrow \infty}|k(t)+a+d| \leq C \sigma, \\
& \limsup _{t \rightarrow \infty}\left|(x(t) y(t)) \exp \left(-R^{T} t\right) \Delta \exp (-R t)\left(\begin{array}{c}
x(t) \\
y(t)
\end{array}\right)+(a+d) \sigma\right| \leq C \sigma^{3 / 2} .
\end{aligned}
$$

Here $C$ is some positive constant independent of $\sigma$. The matrix $R$ is defined in (52).

Remark III.2 In vicw of the fact we can rescale the equations such that $\sigma=1$, see Fact (vi), it is clear that the above Theorem III.1 remains valid 
for $\sigma$ not necessarily small. However, obviously the theorem's conclusions are strongest for small values of $\sigma$.

Remark III.3 Consider $d$ as bifurcation parameter. In conjunction with the observation that a regular Hopf bifurcation takes place for $c+a d>0$ and $d$ passing through $-a$, we conchude from the Theorem III.1 that the Hopf bifurcation is such that as $d$ decreases through $-a$ a stable limit cycle forms whilst the equilibrium becomes unstable. Appendix IV establishes this fact rigorously.

Finally let us provide the details of the calculations for $\Delta$ and $\Gamma$. Recall (52), we have

$$
\exp (R t)=\left(\begin{array}{cc}
\cos (\omega t)-\frac{1}{\omega} \sin (\omega t) & \frac{1}{\omega} \sin (\omega t) \\
-\frac{c}{\omega} \sin (\omega t) & \cos (\omega t)+\frac{1}{\omega} \sin (\omega t)
\end{array}\right) .
$$

It follows that,

$$
\begin{aligned}
& \exp (-R t) b b^{T} \exp (R t)= \\
& \left(\begin{array}{cc}
-\frac{c}{\omega^{2}} \sin ^{2}(\omega t) & -\frac{1}{\omega^{2}} \sin (\omega t)(\omega \cos (\omega t+\sin (\omega t)) \\
-\frac{c}{\omega^{2}}(\omega \cos (\omega t)-\sin (\omega t)) \sin (\omega t) & \cos ^{2}(\omega t)-\frac{1}{\omega^{2}} \sin ^{2}(\omega t)
\end{array}\right),
\end{aligned}
$$

from which we readily deduce the expression for $\Gamma$. Similarly from

$$
\begin{aligned}
& \exp \left(R^{T} t\right) b b^{T} \exp (R t)= \\
& \left(\begin{array}{cc}
\frac{c^{2}}{\omega^{2}} \sin ^{2}(\omega t) & -\frac{c}{\omega^{2}} \sin (\omega t)(\omega \cos (\omega t+\sin (\omega t)) \\
-\frac{c}{\omega^{2}}(\omega \cos (\omega t)+\sin (\omega t)) \sin (\omega t) & \left(\cos (\omega t)+\frac{1}{\omega} \sin (\omega t)\right)^{2}
\end{array}\right),
\end{aligned}
$$

we compute $\Delta$ as the average over one period of the above expression.

\section{Existence and uniqueness of limit cycle}

Consider the adaptive system (18) with $e=0$ and parameters in the rangc $c>a^{2}$ and $d<-a$. Let $\omega^{2}=c-a^{2}>0$ as in Appendix III. Introduce the variables $z_{1}=x, z_{2}=-a x+y, z_{3}=k-d-a$. The system description becomes:

$$
\begin{aligned}
& \dot{z_{1}}=z_{2} \\
& \dot{z_{2}}=-\omega^{2} z_{1}-z_{3}\left(z_{2}+a z_{1}\right) \\
& \dot{z_{3}}=-\sigma z_{3}+\left(z_{2}+a z_{1}\right)^{2}+\sigma(d+a) .
\end{aligned}
$$


Introduce now cylinder co-ordinates, $z_{1}=\sqrt{\sigma} r \sin (\omega \theta), z_{2}=\sqrt{\sigma} \omega r \cos (\omega \theta)$ and $z_{3}=\sqrt{\sigma} z$. Denote $\sqrt{\sigma}=\varepsilon$. This leads to the system description:

$$
\begin{aligned}
\dot{r} & =-\frac{\varepsilon}{\omega} z r(\cos (\omega \theta)+a \sin (\omega \theta)) \sin (\omega \theta), \\
\dot{\theta} & =-1+\frac{\varepsilon}{\omega^{2}} z(\cos (\omega \theta)+a \sin (\omega \theta)) \cos (\omega \theta), \\
\dot{z} & =-\sigma z+\varepsilon\left((d+a)+r^{2}(\cos (\omega \theta)+a \sin (\omega \theta))^{2}\right) .
\end{aligned}
$$

Utilizing Theorem 1.1 pp261 [14], we deduce that there exists $\varepsilon^{*}>0$ such that for all $0<\varepsilon<\varepsilon^{*}$ the system (62) possesses an isolated periodic orbit $\left(r(\theta, \varepsilon), z(\theta, \varepsilon)=\left(\sqrt{\frac{-2(d+a)}{1+a^{2}}}, 0\right)+O(\varepsilon)\right.$. The periodic orbit $(r(\theta, \varepsilon), z(\theta, \varepsilon)$ is continuously differentiable in $\varepsilon$.

The conditions of Theorem 1.1 pp261 [14] are that the equations:

$$
\begin{aligned}
& 0=z_{0} r_{0}, \\
& 0=(d+a)+r_{0}^{2} \frac{\left(1+a^{2}\right)}{2},
\end{aligned}
$$

have a solution for which

$$
\operatorname{det}\left(\begin{array}{cc}
0 & -a \sqrt{\frac{-(d+a)}{2\left(1+a^{2}\right)}} \\
\sqrt{-2(d+a)\left(1+a^{2}\right)} & 0
\end{array}\right)=-a(d+a) \neq 0 .
$$

The latter is ensured by assumption as $a(d+a)<0$.

For the local asymptotic stability of the periodic orbit (in $(r, z)$-space), it suffices to observe that the trace of the Jacobian is $-\sigma<0$. Alternatively, we can exploit the averaging ideas of Appendix III.

From the above we deduce that the system (18) with $a+d<0$ and $c>a^{2}$ possesses a locally asymptotically stable periodic orbit for all sufficiently small $\sigma$ :

$$
\begin{aligned}
x(t) & =-\sqrt{\frac{-2 \sigma(d+a)}{1+a^{2}}} \sin (\omega t)+O(\sigma) \\
y(t) & =\sqrt{\frac{-2 \sigma(d+a)}{1+a^{2}}}(-a \sin (\omega t)+\omega \cos (\omega t))+O(\sigma) \\
k(t) & =-(d+a)+O(\sqrt{\sigma}) \\
\omega^{2} & =c-a^{2}>0
\end{aligned}
$$

\section{How to remove DC offset error}

As is well known, the DC offset error can be removed from the output through integral action. It is probably well less known that the resulting dynamical 
system remains in the same class of systems studied in this paper. Hence our analysis also applies to these systems where integral action is present. Starting from the system description:

$$
\begin{aligned}
P\left(\frac{d}{d t}\right) y & =Q\left(\frac{d}{d t}\right) u, \\
\frac{d}{d t} y_{I} & =y, \\
u & =-k\left(y+\alpha y_{I}\right)+e .
\end{aligned}
$$

Here $\alpha>0$ is the relative integral action applied in the control and $y_{I}$ is the integral of $y$. Considering a new output $\eta=y+\alpha y_{I}$ we can equivalently represent the control system as:

$$
\begin{aligned}
\frac{d}{d t} P\left(\frac{d}{d t}\right) \eta & =\left(\frac{d}{d t}+\alpha\right) Q\left(\frac{d}{d t}\right) u \\
u & =-k \eta+e \\
\left(\frac{d}{d t}+\alpha\right) y & =\frac{d}{d t} \eta .
\end{aligned}
$$

This system (67) is clearly of the same form as the system (1). We can now procecd as in the rest of the paper, with the added flexibility of using $y$ and/or $\eta$ in the adaptive law that defines $k$. The benefit of the integral action is that whenever the adaptive system regulates to an equilibrium that then the output $y$ is regulated to zero. Clearly this is important from a control perspective, but it does not alter in any significant sense the overall dynamics of the adaptive system. Moreover as regulation to an equilibrium is by no means guaranteed, nor the most likcly cvent, integral action looses its attraction.

\section{Acknowledgments}

Iven Marecls acknowledges the School of Mathematical Sciences, Exeter for its support for his visit in 1997, during which this paper was initiated and the support of NWO for his visit in January 1999 to the University of Twente, during which period this paper was finalised. Achim Ilchmann acknowledges the support received by the University of Melbourne for his visit to the Department of Electrical and Electronic Engineering in 1998.

\section{References}

[1] J.W.Polderman, J. C. Willems, An Introduction to Mathematical Systems Theory: A Behavioral Approach, Springer, Berlin 1998.

[2] I. Mareels and J.W. Polderman, Adaptive Systems: An Introduction, Birkhäuser, Boston, 1996. 
[3] I. Mareels, A simple selftuning controller for stably invertible systems Systems \& Control Letters, Vol 4, pp5-16, Feb.1984.

[4] A. Ilchmann Non-Identifier-Based High-Gain Adaptive Control, SpringerVerlag, London, 1993.

[5] A. Ilchmann, Adaptive $\lambda$-tracking for polynomial minimum phase systems; Dynamics and Stability of Systems Vol 13, pp341-371, 1998.

[6] A. Ilchmann, E.P. Ryan, Universal $\lambda$-tracking for nonlinearly-perturbed systems in the presence of noise, Automatica Vol 30, pp 337-346, 1994.

[7] B. Mårtensson, J.W. Polderman, Correction and simplification to: The order of a stabilizing regulator is sufficient a priori information for adaptive stabilization, Systems \& Control Letters, Vol 20, pp. 465-470, 1993.

[8] P. Ioannou, P.V. Kokotović, Instability analysis and improvement for robustness of adaptive control, Automatica Vol 20, pp 583-594, 1984.

[9] J.A. Sanders, F. Verhulst, Averaging methods in nonlinear dynamics, Springer verlag, Applied Mathematical Sciences, Vol 59, New York 1985.

[10] C.I. Byrnes, J.C. Willems, Global Adaptive Stabilization in the Absence of Information on the Sign of the High Frequency Gain. Part I: Analysis and Optimization of Systems. In: Proc. 6th Intern. Conf. on Anal. and Optimiz. of Syst., A. Bensoussan and J.L. Lions Eds. Springer Verlag, pp 49-57, New York, 1984.

[11] H. Kaufman H., I. Bar-Kana, K. Sobel, Direct Adaptive Control Algorithms:Theory and Applications, Springer-Verlag, New York, 1994.

[12] A. S. Morse, Recent problems in parameter adaptive control, in Outils et Modèles Mathématiques pour l'Automatique, l'Analyse de Systèmes et le Traitment du Signal, I.D. Landau, Ed., pp.733-740 (Editions du CNRS 3, Paris)

[13] Y. Kuznetsov, Elements of applied bifurcation theory, 2nd ed, Applied Mathematical Sciences, Vol 112, Springer, New York 1998.

[14] J.K. Hale, Ordinary Differential Equations, 2nd ed, Krieger, Malabar Florida, 1980 